\title{
A Method to Determine Thermal Conductivity using Boundary Temperature Measurements
}

\author{
JUKKA MYLLYMÄKI and DJEBAR BAROUDI \\ VTT Building Technology, Fire Technology \\ P.O. Box 1803, FIN-02044 VTT, FINLAND
}

\begin{abstract}
A numerical method is presented to determine the thermal conductivity of homogeneous insulation material using boundary temperature measurements. In this inverse analysis the direct problem consists of non-linear partial differential equation that is semidiscretized via the variational form of the heat conduction problem. The inverse problem is reduced to minimization of a regularized functional of residuals solved by numerical gradient methods. Tikhonov and mesh coarsing regularizations are used. This method is known as the Regularized Output Least Squares Method (RLS). The accuracy of the predicted results is examined from an illustrated 1-D case of a fire protected steel plate. Transient test temperature of the steel plate is simulated. Noise of randomly varying amplitude is added to the simulated temperature. Results show that good estimations on the thermal conductivity can be obtained without measuring temperature inside the insulation material. Comparisons with the accuracy of technique used in methods NT FIRE 021 and Pr ENV YYY5-4 of fire protected steel structures are presented.
\end{abstract}

KEYWORDS: inverse methods, heat conduction, thermal conductivity, fire protection

\section{NOMENCLATURE}

C capacitance matrix $\left(\mathrm{J} \mathrm{m}^{-2} \mathrm{~K}^{-1}\right)$ $C_{i j}^{e}$ elements of the capacitance matrix $\left(\mathrm{Jm}^{-2} \mathrm{~K}^{-1}\right)$

$c$ specific heat $\left(\mathrm{J} \mathrm{kg}^{-1} \mathrm{~K}^{-1}\right)$

f heat flux (force) vector $\left(\mathrm{W} \mathrm{m}^{-2}\right)$

$h$ convection coefficient $\left(\mathrm{W} \mathrm{m}^{-2} \mathrm{~K}^{-1}\right)$

$\mathbf{K}$ conductance matrix $\left(\left(\mathrm{W} \mathrm{m}^{-2} \mathrm{~K}^{-1}\right)\right.$

$l^{e}$ element length $(\mathrm{m})$
$N_{i} \quad i:$ th interpolation (shape) function

$\mathbf{N}^{e}(x)$ vector of element shape functions

$N_{i, \xi}$ derivative of interpolation function $i$

$q$ heat flux $\left(\mathrm{Wm}^{-2}\right)$

$r \quad$ source term $\left(\mathrm{Wm}^{-3}\right)$

$\mathbf{T} \quad$ vector of nodal temperatures $(\mathrm{K})$

$\mathbf{T}^{e} \quad$ vector of element nodal temperatures 


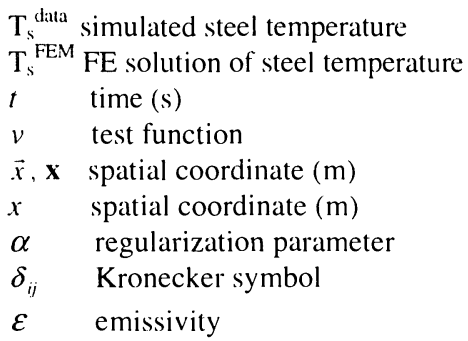

$T_{s}^{\text {data }}$ simulated steel temperature

$\lambda \quad$ thermal conductivity $\left(\mathrm{W} \mathrm{m}^{-1} \mathrm{~K}^{-\mathrm{i}}\right.$ )

$\lambda_{s} \cdot \lambda_{p}, \quad$ conductivity of steel and protection

$\rho \quad$ density $\left(\mathrm{kg} \mathrm{m}^{-3}\right)$

$\sigma \quad$ Stefan-Boltzmann constant. $5.67 \times 10^{-8} \mathrm{Wm}^{-2} \mathrm{~K}^{-1}$

$\Omega, \Omega^{c} \quad$ global and element solution domain

FE, FEM Finite Element Method

ODE Ordinary Differential Equation

1-D, 2-D one and two dimensional space

\section{INTRODUCTION}

To date various methods have been developed for analysis of the inverse heal conduction problem involving the determination of thermal conductivity from measured temperatures inside the material [1-10]. Usually the inverse solution is based on the minimization of the differences between measured and calculated temperatures in a least squares sense [1-9]. This method is called the Output Least Squares Method (OLS). Existing methods of inverse analysis $[1-4,8-10]$ require multiple spatial temperature measurements inside the material. Many thermocouples should be used to obtain accurate results in such methods. More thermocouples do demand more cost and time. The computational experiments have shown that the accuracy of reconstruction of thermal conductivity depends critically upon the location of thermocouple [10]. When only boundary measurements are performed, the material does not need to be drilled for the mounting of thermocouples to measure internal temperatures, and the errors due to the location errors of thermocouples are avoided. To date only a few studies have studied the inverse determination of thermal conductivity from boundary measurements [5-7]. Kohn and Vogelius [6] determined thermal conductivity from static boundary measurements with temperature non-dependent thermal conductivity. Huang and co-workers [5] applied the conjugate gradient method of minimization using two thermocouples located on the boundary surfaces. Lin and Cheng [7] investigated the hybrid Laplace transform/control volume technique in determining temperature-dependent thermal conductivity of a homogeneous material from boundary temperature measurements.

Methods suggested for use in connection with fire testing have been reviewed by Lundqvist et al. [8]. Dhima [9] has used OLS with finite difference discretization for the determination of thermal properties of fire protections using plate type of test specimens. Within the field of fire science testing methods have been developed based on one differential equation for fire protections of steel structures $[11,15,16]$.

A common feature of inverse problems is the instability, that is, small changes in the data may give rise to large changes in the solution. Small finite dimensional problems are typically stable, however, as the discretization is refined, the number of variables increases and the instability of the original problem increases. Therefore regularization is needed. Two regularization methods, mesh coarsing and Tikhonov-regularization, have been adopted in order to get a stabilized solution. The available a priori known physical constraints on the parameters are taken into account in the minimization. 
The aim of this study is to investigate the feasibility of finite element technique combined with regularized output least squares method in determining temperature-dependent thermal conductivity of a homogeneous insulation material from boundary temperature measurements. Thermal conductivity is approximated as piece-wise linear functions of temperature. The unknown values of thermal conductivity at different temperatures are found by minimizing a constrained and regularized functional. The functional consists of the sum of the squares of residual norm of the errors (data - model) plus the square of the norm of the second derivatives of the properties with respect to the temperature. An appropriate balance between the need to describe the measurements well and the need to achieve a stable solution is reached by finding an optimal regularization parameter. Both Newton and conjugate gradient methods have been used in the minimization. The Morozov discrepancy principle is used to find a reasonable value for the regularization parameter.

To investigate the accuracy of the present inverse method in estimation of thermal conductivity from boundary temperature measurements a simulated 1-D case example of a fire protected steel sheet is investigated. The effects of the spatial discretization of the finite elements and temperature measurement noise to the accuracy of the inverse solution are studied. Comparisons with the standard differential equation method used for fire protected steel structures in NT FIRE $021[15]$ and Pr ENV YYY5-4 [16] are made.

\section{FORMULATION OF THE DIRECT PROBLEM (HEAT CONDUCTION PROBLEM)}

The basic idea is to solve the temperature field $T(x, t)$ in a given material region. The field equation

$$
\rho c \dot{T}(\vec{x}, t)=\vec{\nabla} \cdot(\lambda \vec{\nabla} T(\vec{x}, t))+r(t, \vec{x}, T)
$$

is the diffusion equation with $r(t, x, T)$ as an arbitrary source term. Time derivatives are indicated by superimposed dots $(\partial T / \partial t=\dot{T})$. The Fourier heat conduction constitutive relation is assumed. This equations is complemented with the appropriate initial-boundary conditions to get a well-posed problem. The boundary conditions may be a Dirichlet type or Neumann type normal heat flux $q_{n}=h(T)\left(T-T_{\infty}\right)+\sigma \varepsilon\left(T^{4}-T_{\infty}^{4}\right)$ with convection and radiation parts. The boundary terms as also the source terms if present will be included into the nodal flux vector of the discretized heat conduction equation.

Using the standard finite element semidiscretization [12] one obtains the variational form of the problem (1) as

$$
\int_{\Omega} \rho c \dot{T} v d \Omega+\int_{\Omega} \lambda \vec{\nabla} T \cdot \vec{\nabla} v d \Omega=\int_{\Omega} r v d \Omega-\int_{\delta_{2} \Omega_{q}} \vec{q} \cdot \vec{n} v d \Gamma
$$

with the temperature field approximated by $T^{c}(x, t)=\mathbf{N}^{c}(x) \mathbf{T}^{c}(t)$, where the test and the basis functions $N_{i}(\xi)$ are the same (Galerkin formulation). In the case of 1-D for a linear element 
we have $N_{1}(\xi)=(1-\xi) / 2$ and $N_{2}(\xi)=(1+\xi) / 2$, where $\xi=x / 1$ is the local element coordinate.

The semi-discretization of the heat conduction equation (2) produces the non-linear initial value problem

$\mathbf{C}(t, \mathbf{T}) \dot{\mathbf{T}}(t)=\mathbf{f}(t, \mathbf{T})-\mathbf{K}(t, \mathbf{T}) \mathbf{T}(t), t>0$

$\mathbf{T}(0)=\overline{\mathbf{T}}_{0}, \quad t=0$,

where $\mathbf{T}(\mathrm{t})$ is the global vector of the unknown temperatures. Equation (3) is a set of $n \times l$ non-linear ordinary differential equations. Notice that the right hand in the equation (2) corresponds to the nodal flux vector $\mathbf{f}(t, \mathbf{T})$, which contains the boundary terms. Equation (3) is to be complemented with appropriate initial conditions. Natural boundary conditions are already included in the variational form (2). The essential boundary conditions are taken into account during the solution process of the initial value problem. The global matrices and vectors are assembled using standard FE-assembling techniques. In 1-D case the following matrices; the element conductance matrix

$K_{i j}^{e}=\int_{\Omega^{*}} \lambda(T(\mathbf{x})) \vec{\nabla} \mathrm{N}_{\mathrm{i}}(\mathbf{x}) \cdot \vec{\nabla} \mathrm{N}_{\mathrm{j}}(\mathbf{x}) d \Omega=\frac{2}{l^{e}} \int_{-1}^{1} \lambda(T(\xi)) N_{i, \xi}(\xi) N_{j . \xi}(\xi) d \xi$

the element capacitance matrix

$C_{i j}^{e}=\int_{\Omega^{e}} \rho(T(\mathbf{x})) c(T(\mathbf{x})) \mathrm{N}_{\mathrm{i}}(\mathbf{x}) \mathrm{N}_{\mathrm{j}}(\mathbf{x}) d \Omega=\frac{l^{e}}{2} \int_{-1}^{1} \rho(T(\xi)) c(T(\xi)) N_{i}(\xi) N_{j}(\xi) d \xi$

and the element nodal flux vector

$f_{i}^{e}=-\int_{\partial \Omega_{q} \cap \partial \Omega^{e}} \vec{q} \cdot \vec{n} \mathrm{~N}_{\mathrm{i}}(\mathbf{x}) d \Gamma=-\left[q_{n} N_{i}(\xi)\right]_{\partial \Omega_{q} \cap \partial \Omega^{e}}$

are obtained. The element matrices and vectors are integrated numerically using GaussLegendre integration scheme. The element matrices and vectors depend on the unknown temperature. In the present analysis the explicit forward Euler time integration scheme has been used. The Gauss-Legendre integration of Eq. 5 leads to consistent capacitance matrix, where the non-diagonal terms $C_{i j}^{e}(i \neq j)$ are non-zero. Here Newton-Cotes integration formula where the nodal points are used as integration points and the weights of the integration are calculated as $w_{i}=\int_{0}^{1} N_{i} N_{i} d x$, has been used in order to get a diagonal capacitance matrix.

\section{FORMULATION OF THE INVERSE HEAT CONDUCTION PROBLEM (IHCP)}

Consider determination of temperature dependent thermal properties in an initial value problem (3) using measured temperature. Unknown thermal conductivity is discretized with respect to 
the temperature using piece-wise linear basis functions. Vector $\mathbf{a}=$ $\vec{a}(\mathbf{T})=\left[\lambda\left(T_{1}\right) \ldots \lambda\left(T_{i}\right), \lambda\left(T_{i+1}\right) \ldots \lambda\left(T_{M}\right)\right]$ contains nodal values of the unknown thermal conductivity at temperature interval $\left[T_{1}, T_{M}\right]$, where $T_{1}$ and $T_{M}$ are the minimal and the maximal values of temperature in solution domain. A realistic initial value is given for a. Using the regularized output least squares method (RLS) |13| a is solved from the minimization problem.

$\min _{\text {iieD }}\left\{\left\|T^{\text {FEM }}(\vec{a} ; \vec{x} ; t)-T^{\text {deltu }}(\vec{x}, t)\right\|^{2}+\alpha\|\mathbf{L} \vec{a}\|^{2}\right\} \quad$ with respect to $\mathbf{a}$.

where vector $T^{\text {FEM }}(\vec{a} ; \vec{x} ; t)=\mathbf{N}(\vec{x}) \mathbf{T}(t)$ is the solution of the direct initial value problem (3). Vector $T^{\text {data }}$ contains the input data; the real measured temperatures or simulation of them. Euclidean norms are calculated in the temperature measurement points $x_{i}$ at temperature sampling time $t_{j}$ as $\|f(\vec{x} ; t)\|^{2}=\sum_{i} \sum_{j}\left|f\left(x_{i} ; t_{j}\right)\right|^{2}$.

Since the inverse problem is ill-posed, i.e. smail variations in the temperature measurements cause large scatter in the inversion results, it has to be regularized. In RLS method one seeks a minimum for the functional (6) where $\alpha(>0)$ is a small regularization parameter and differential operator $\mathbf{L}=\mathbf{I}, d \mathbf{a} / d T$ or $d^{2} \mathbf{a} / d T^{2}$. Parameter $\alpha$ controls how much weight is given to the residual norm $\left\|T^{F E M}(\vec{a} ; \vec{x} ; t)-T^{\text {datu }}(\vec{x}, t)\right\|$ enforcing the consistency relative to the norm $\|\mathbf{L} \vec{a}\|$ enforcing stability of the solution. The problem is the appropriate choice of the parameter $\alpha$ so that we can distinguish the real signal from the measurement noise. Perhaps clearest rule to choose the regularization parameter is Morozov discrepancy principle [10, 13] where the residual norm is set equal to upper bound

$\left\|T^{\text {FEM }}(\vec{a} ; \vec{x} ; t)-T^{\text {detu }}(\vec{x}, t)\right\|^{2}=R \delta^{2}$,

where $\delta^{2}$ is measure of noise during time $\delta^{2}=\int_{0}^{t \max }|\partial|^{2} d t$ and $\partial$ is the amplitude of noise at certain time and $R$ is a coefficient of value $1.6-1.7$.

\section{NUMERICAL EXPERIMENTS}

\section{Basic Data of the Studied Example}

To assess the accuracy of the present numerical algorithm in predicting the unknown thermal conductivity, 1-dimensional case of an insulated steel plate is considered (Fig. 1). Following material parameters have been used; density of steel $\rho_{s}=7850 \mathrm{~kg} / \mathrm{m}^{3}$, specific heat of steel $c_{s}=540 \mathrm{~J} \mathrm{~kg}^{-1} \mathrm{~K}^{-1}$, density of fire protection $\rho=220 \mathrm{~kg} / \mathrm{m}^{3}$, specific heat of fire protection $c_{p}=1000 \mathrm{~J} \mathrm{~kg}^{-1} \mathrm{~K}^{-1}$. The thickness of the steel plate is assumed to be $d_{s}=0.0041 \mathrm{~m}$ and the 
thickness of the fire protection is $d_{n}=0.02 \mathrm{~m}$. As the thermal conductivity of the steel a value $\lambda_{s}=50 \mathrm{Wm}^{-1} \mathrm{~K}^{-1}$ has been applied.

The present example investigates the inverse problem where the exact function of the thermal conductivity of the fire protection is following

$\lambda_{p}(T)=\lambda_{o}\left(1+\left(\frac{T}{T_{\lambda}}\right)^{p_{\lambda}}\right)$,

where $\lambda_{o}=0.0251 \mathrm{~W} \mathrm{~m}^{-1} \mathrm{~K}^{-1}, T_{\lambda}=411 \mathrm{~K}, p_{\lambda}=2.404$ and $T$ is temperature of the fire protection.

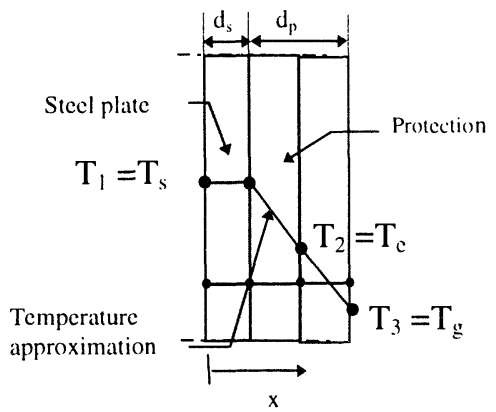

FIGURE 1. Insulated steel plate with fire protection divided into two elements.

\section{Calculation of Fictitious Test Data}

A simulated numerical approximation of exact solution has been obtained by solving the direct problem by finite element program ABAQUS Version 5.4 [14] by using the values given above and the known thermal conductivity defined in Eq. (8). The solution domain was divided into 13 one dimensional 2-noded diffusive heat transfer link elements, element type DC1D2, with three elements in the steel part with element length $l^{c}=0.001367 \mathrm{~m}$ and 10 elements in the insulation part with element length $l^{e}=0.002 \mathrm{~m}$. In all the first order elements of ABAQUS the internal energy storage term associated with specific heat was integrated at the nodes.

The boundary condition at $x=0$ has been assumed to be adiabatic, i.e. $q=0$. The temperature at the boundary of the fire protection $x=d_{s}+d_{p}$, has been assumed to follow the standard ISO 834 temperature given in Fig. 2. The initial temperature $T_{0}$ was assumed to be $20{ }^{\circ} \mathrm{C}$. 


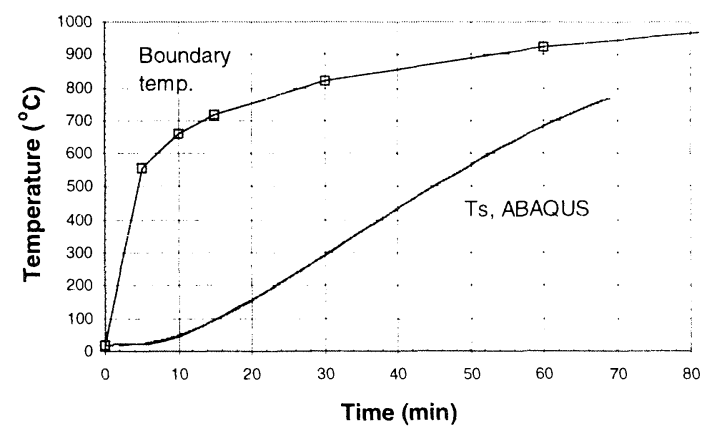

FIGURE 2. Boundary temperature and ABAQUS solution of the steel temperature.

Simulated measured temperature data of the steel used as data was calculated from equation

$T_{s}^{\text {data }}\left(t_{i}\right)=T_{s}^{A B A}\left(t_{i}\right)+k_{i} \delta T$

$t_{i}=i \Delta t_{c o l}$

where $T_{s}^{A B A}(t)$ is the temperature of the steel plate calculated with ABAQUS and $\delta T$ represents an amplitude of simulated temperature measurement noise and $k_{i}$ is a generated random variable. The values of $k_{i}$ lies in the range from -1 to 1 . The data sampling time-step has been $\Delta t^{\prime \prime \prime l l}=120 \mathrm{~s}$.

\section{Assumptions of the Inverse Computation}

In the inverse computation the steel plate was divided into a one lumped element with constant temperature field. This causes that the steel is only acting by specific heat. The fire protection was divided into several elements of equal length. Inverse solution of thermal conductivity was obtained by minimizing the equation

$\min _{\vec{a} \in D}\left\{\left\|T_{s}^{F E M}\left(\vec{a} ; t_{i}\right)-T_{s}^{d d a t a}\left(t_{i}\right)\right\|^{2}+\alpha\|\mathbf{L} \vec{a}\|^{2}\right\}$

where $T_{s}^{\text {FEM }}$ is the steel temperature obtained as a solution of direct initial value problem (3) and $T_{s}^{\text {datu }}$ is the simulated temperature of the steel which was computed from Eq. (9). The "unknown" thermal conductivity of the fire protection $\lambda_{p}(T)$ was divided into a certain number of sub-intervals $\left[T_{i}, T_{i+1}\right]$ of length $50{ }^{\circ} \mathrm{C}$. It was assumed that we know the exact value of 
the thermal conductivity at $20{ }^{\circ} \mathrm{C}$ as we usually do in the practice. The unknown vector $\vec{a}^{T}=\left[\lambda_{p}(50), \lambda_{p}(100), \lambda_{p}(150) \ldots\right]$ consisted of the values of thermal conductivity at temperatures $50{ }^{\circ} \mathrm{C} \ldots 900{ }^{\circ} \mathrm{C}$. Positiveness of thermal conductivity $\lambda_{p}(T)>0$ was used also as restriction to the admissible solution. As an initial value for each element of vector $i^{T}$ at the start of each iteration the known value of thermal conductivity at $20{ }^{\circ} \mathrm{C}$ was used.

\section{The Effect of Spatial Discretization to the Accuracy of Inverse Solution}

The effect of the spatial discretization to the accuracy of the solution was studied by changing the number of elements in the fire protection used in the inverse solution from one to seven. The value $\delta T=1{ }^{\circ} \mathrm{C}$ was used as an amplitude of simulated temperature measurement noise in the Eq.(12) when calculating simulated measured data.

It can be seen in Fig. 3a that the error between the real thermal conductivity and thermal conductivity obtained by inversion with one element at temperatures $50{ }^{\circ} \mathrm{C}-350{ }^{\circ} \mathrm{C}$ is quite large. This error is due to the linear assumption and the fact that only one Gaussian integration point is used in the middle of the element. When temperature at the boundary of the insulation rises quite rapidly the temperature at the center of the insulation calculated with one element is much higher than the correct one (see Fig. 6a). Due to this the inverse solution is also incorrect.

TABLE 1. Dependence of the relative error $\operatorname{Err}(\%)$ of $\lambda$ on the number $\mathrm{N}^{\mathrm{c}}$ of elements

\begin{tabular}{lr}
\hline $\mathrm{N}^{\mathrm{e}}$ & $20-900{ }^{\circ} \mathrm{C}$ \\
\hline 1 & 31.18 \\
2 & 2.97 \\
3 & 1.77 \\
4 & 1.63 \\
5 & 1.73 \\
7 & 1.60 \\
\hline NT 021/prENV YYY5-4 & 77.98 \\
\hline
\end{tabular}

Already with two elements the inverse solution is rather satisfactory (Fig. 3b). The convergence of the inverse solution can be seen in Fig. $4 \mathrm{a}$ where the error of the thermal conductivity is plotted as a function of the number of elements $N^{*}$. Here the error of the thermal conductivity in certain temperature interval $\left[T_{1}, T_{M}\right]=\left[20{ }^{\circ} \mathrm{C}, 900{ }^{\prime \prime} \mathrm{C}\right]$ has been computed using following relative error measure:

$\operatorname{Err}(\%)=\frac{100}{T_{M}-T_{1}} \int_{T_{1}}^{T_{M}} \frac{\left|\lambda_{e x}(T)-\lambda_{e s}(T)\right|}{\left|\lambda_{e x}(T)\right|} d T$

where the subscripts es and ex denote the estimated and exact values, respectively. The calculated errors are also shown in Table 1. It can be seen that the inverse solution converges to the 
correct solution as the number of elements is increased. This was observed also by Lin and Cheng in their inversion method where they increased the number of control volumes [7].

\section{Accuracy of Standard Methods of Fire Technology}

In the method NORDTEST NT FIRE $021 \mid 15]$ and in the corresponding CEN standard prENV YYY5-4 [16] of fire protection to steel structures thermal conductivity is calculated using inverse solution of the differential equation derived by Wickström [11] using the measured steel and gas temperatures. Thermal conductivity calculated using the method NT FIRE 021/prENV YYY5-4 is presented in Fig. 4b. Corresponding error is shown in Table 1.
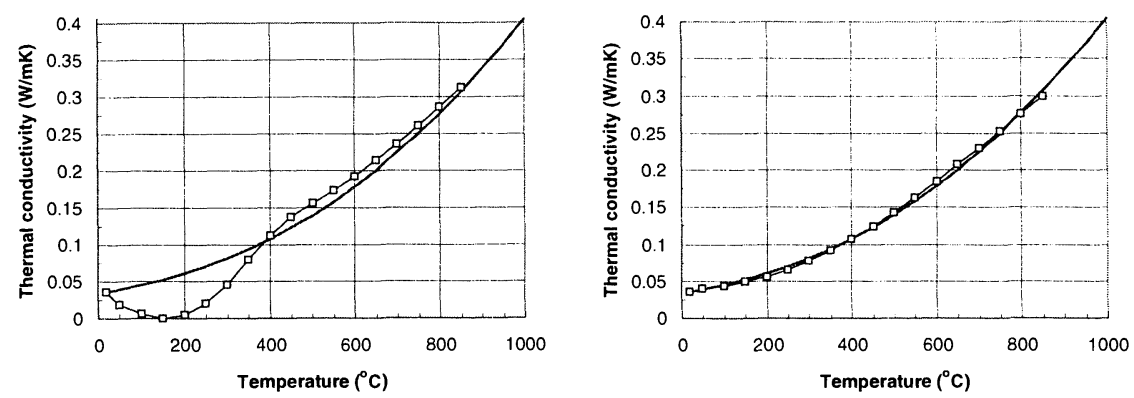

a)

b)

FIGURE 3. Inverse solution of the thermal conductivity (line with squares) with a) 1 -element b) two elements and compared to the exact thermal conductivity (solid line).

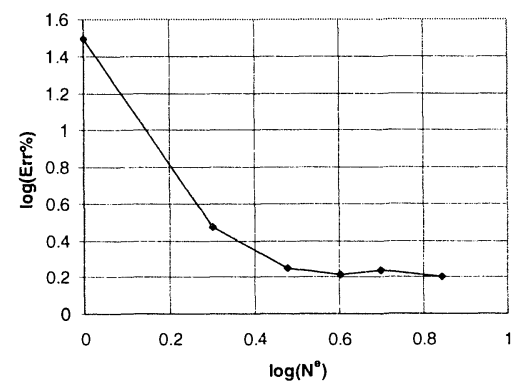

a)

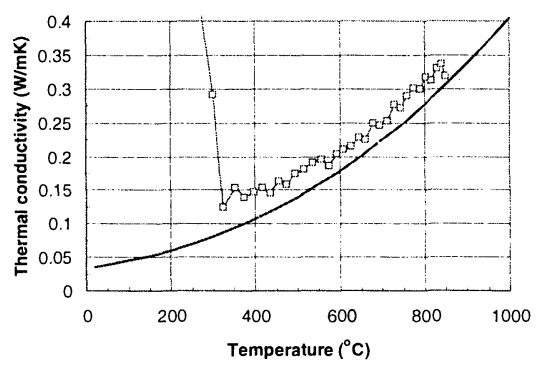

b)

FIGURE 4. a) Convergence of the inverse solution of the thermal conductivity as a function of the number of elements, b) Inverse solution of the thermal conductivity (line with squares) by using the method NT FIRE $021 /$ prENV YYY5-4, measurement error $\delta T=1{ }^{\circ} \mathrm{C}$ 


\section{Effect of Noise in Temperature Data}

The effect of the noise in temperature data to the accuracy of the solution was studied by changing the amplitude of the noise in the limits $\delta T=1{ }^{\circ} \mathrm{C} \ldots 10{ }^{\circ} \mathrm{C}$ in the Eq. (12) when calculating simulated data. In the Table 2 , the errors in the thermal conductivity obtained by inversion are presented. In the regularized case (RLS), Morozov discrepancy principle (7) has been applied as restraint when solving Eq. (10) keeping the residual constant $\left\|T_{s}^{\text {FEM }}\left(\vec{a} ; t_{i}\right)-T_{s}^{\text {ddatu }}\left(t_{i}\right)\right\|^{2}=R n \delta T^{2}$, where $n$ is the number of time steps. The non-regularized $(\alpha=0)$ case is also presented in Table 2. This corresponds the Output Least Squares Method (OLS). It can be seen that the increase of noise does not affect much the accuracy of the RLS method. Using OLS the increase of noise causes large errors to the inverse solution. The results are plotted in Fig.5a.

TABLE 2. Average relative error $\operatorname{Er}(\%)$ of $\lambda$ with different amplitudes of noise $\delta T$ with (RLS) and without (OLS) regularization

\begin{tabular}{rrr}
\hline$\delta T$ & $20-900{ }^{\circ} \mathrm{C}$ & $20-900{ }^{\circ} \mathrm{C}$ \\
$\left({ }^{\circ} \mathrm{C}\right)$ & $(\mathrm{RLS})$ & $(\mathrm{OLS})$ \\
\hline 1 & 1.73 & 6.51 \\
2 & 1.38 & 12.69 \\
5 & 2.45 & 36.56 \\
10 & 1.47 & 57.58 \\
\hline
\end{tabular}

\section{Accuracy of Direct Solution Using Thermal Conductivity Computed by Inversion}

The convergence of the direct solution can be seen in Fig. 5b where the error of the calculated temperature field is plotted as a function of the number of elements. Here the error of the temperature field has been computed using following relative error Erl of solution in space Ll and $E r 2$ of solution in space L2

$$
\begin{aligned}
& E r l=\sum_{i}^{N} \sum_{j}^{n}\left|T^{A B A}\left(x_{i}, t_{j}\right)-T^{F E M}\left(x_{i}, t_{j}\right)\right| /\left|T^{A B A}\left(x_{i}, t_{j}\right)\right| \\
& E r 2=\sqrt{\sum_{i}^{N} \sum_{j}^{n}\left\{T^{A B A}\left(x_{i}, t_{j}\right)-T^{F E M}\left(x_{i}, t_{j}\right)\right\}^{2}} / \sqrt{\sum_{i}^{N} \sum_{j}^{n}\left\{T^{A B A}\left(x_{i}, t_{j}\right)\right\}^{2}}
\end{aligned}
$$

where $T^{A B A}$ denotes the temperature in the insulation computed with ABAQUS using 10 elements and exact thermal conductivity. Temperature $T^{\text {FEM }}$ is the direct solution using $1 \ldots 5$ elements and with thermal conductivity computed by inversion. The solution of temperature field $T\left(x_{i}, t_{j}\right)$ has been calculated at the nodal points of grid of 10 elements. It can bee seen in Figure $6 \mathrm{~b}$ that the direct solution is rather good already with two elements.

The convergence of the finite element solution is shown to be dependent on the number of elements in following way $\left\|T^{e x}-T^{F E M}\right\|_{E} \leq C\left(N^{e}\right)^{-\beta}$, where \|\|$_{E}$ is energy norm. If this equation is assumed to be valid also for the "norms" applied here the rate of the convergence $\beta$ can 
be obtained as a slope of the error lines drawn in $\log$-log scale in Fig. 5b. Numerical values of the slopes $\beta$ are 1.80 in $\mathrm{L} 1$ space line and 1.96 in $\mathrm{L} 2$ space line. These values are quite near the proven theoretical value 2 for linear heat conduction problems $[12\}$.
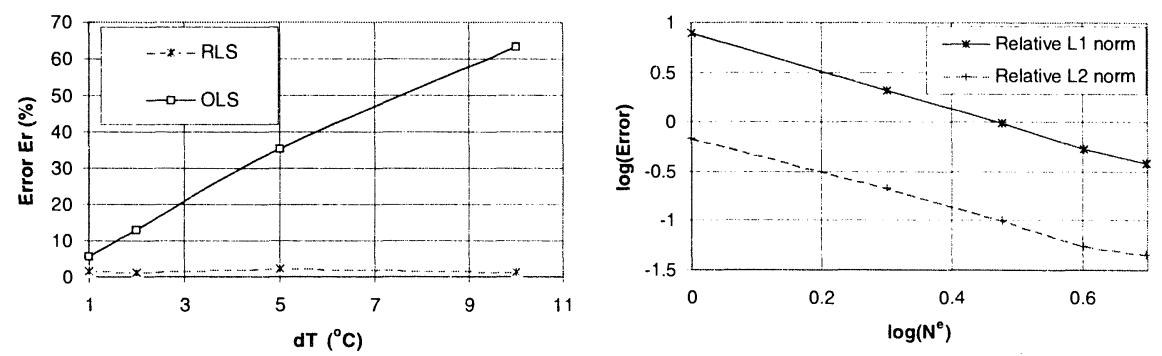

a)

b)

FIGURE 5. a) Average relative error of $\lambda \mathrm{Er}(\%)$ as a function of measurement noise amplitude $\delta T$, b) convergence of the direct solution using conductivity computed by inversion.
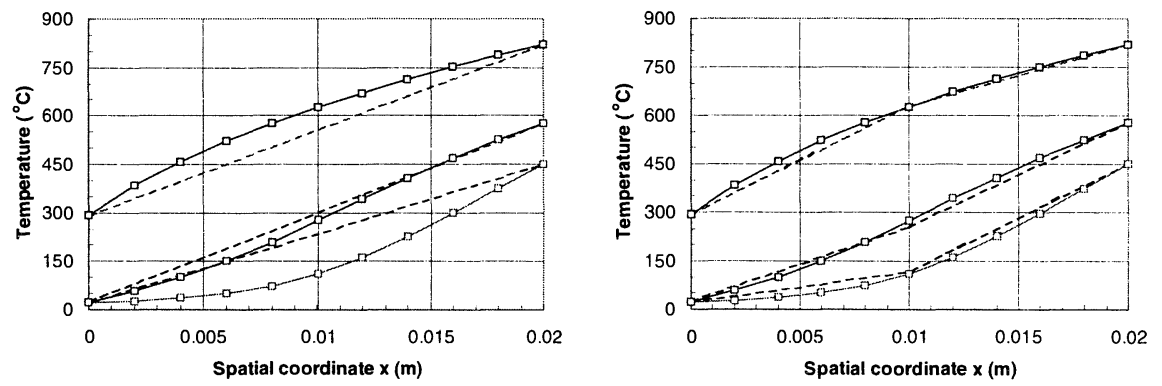

a)

b)

FIGURE 6. Temperature in the fire protection calculated with the inverse solution of the conductivity (dashed line) compared to the ABAQUS solution with exact conductivity (solid line with squares). Solutions with a) 1 -element b) two elements at times $4 \mathrm{~min}, 6 \mathrm{~min}$ and $30 \mathrm{~min}$.

\section{CONCLUSIONS}

A numerical method involving finite element method combined with regularized output least squares method in determining temperature-dependent thermal conductivity of a homogeneous insulation material from boundary temperature measurements is presented. As a numerical experiment insulated steel plate is considered. The effects of element discretization and noise in the temperature data to the accuracy of the inverse solution are studied. The temperature noise is simulated by stochastic values of different amplitude. It is shown that the thermal 
conductivity of the insulation can be computed by inversion in one dimensional case with very good accuracy. Only temperature measurements at the boundaries; in steel and at the surface of the insulation and the adiabatic flux boundary condition at steel surface are used. It is shown that accuracy of the presented method is better than the accuracy of the standard methods of fire protected steel structures. It is also demonstrated that the solution of the temperature field computed with the thermal conductivity obtained by inversion converges towards the exact one.

The presented mathematical inversion method can be extended to two-dimensional cases using two dimensional elements or for cylindrical structures using axisymmetric elements. Extensions of the method to problems where both temperature dependent specific heat and thermal conductivity are unknown are also possible.

\section{REFERENCES}

1. Beck, J. V. and Arnold K.J., Parameter Estimation in Engineering and Science, Wiley, New York, 1977.

2. Alifanov O.M. and Mikhailov V.V., "Solution of the Nonlinear Inverse Thermal Conductivity Problem by the Iteration Method", J. Eng. Phys., 35, 1501-1506, 1978.

3. Tervola P.,"A Method to Determine the Thermal Conductivity from Measured Temperature Profiles", Int. J. Heat Mass Transfer, 32, 1425-1430, 1989.

4. Lam, T.T. and Yeung, W.K., "Inverse Determination of Thermal Conductivity for One-Dimensional Problems", J. Thermophys. Heat Transfer , 9, 335-344, 1995.

5. Chen, H.T., Lin, J.Y., Wu, C.H., and Huang C.H.,"Numerical Algorithm for Estimating TemperatureDependent Thermal Conductivity", Numer. Heat Transfer Part B, 29, 509-522, 1996.

6. Kohn, R. and Vogelius, M.,"Determining Conductivity by Boundary Measurements", Commun. Pure Appl. Math.,37, 289-298, 1984.

7. Lin, J.H. and Cheng T.F.,"Numerical Estimation of Thermal Conductivity from Boundary Temperature Measurements". Numer. Heat Transfer Part A, 32, 187-203, 1997.

8. Lundqvist, A., Mårtensson, U. and Persson, B. 1991., "Measurement of thermal diffusivity in materials at high temperatures". Borås, Sweden, SP REPORT 1991:53. 30 p.1991.

9. Dhima, D. 1986. "Contribution á la charactérisation thermique de matériaux de protection de profilés métalliques soumis á un incendie", Thése de doctorat de l'Université Pierre et Marie Curie, 17.9.1986.

10. Alifanov, O.M. Inverse Heat Transfer Problems, pp. 291-323, Springer-Verlag, Berlin, 1994.

11. Wickström, U. "Temperature Analysis of Heavily-insulated Steel Structures Exposed to Fire", Fire Safety Journal, 9, 281-285, 1985.

12. Eriksson, K., Estep, D., Hansbo P. and Johnson C., Computational Differential Equations. Studentlitteratur, Lund, 1998.

13. Groetsch, C.W. Inverse Problems in the Mathematical Sciences, Vieweg, Braunschweig, 1993.

14. ABAQUS Standard User's Manual Volume I Version 5.4, Hibbit, Karlsson \& Sorensen, Inc. 1994.

15. NT FIRE 021. 1985. "Insulation of steel structures: Fire Protection". NORDTEST 1985.

16. Pr ENV YYY5-4. 1998." Test method for determining the contribution to the fire resistance of structural members: applied protection to steel members". CEN TC $127 \mathrm{doc} 1282$. 